\title{
Evaluación de la accesibilidad espacial a los puestos de la campaña de vacunación antirrábica en Bogotá, Colombia
}

\author{
Stefany Monsalve ${ }^{1,2}$, Daniel Santiago Rucinque ${ }^{1,2}$, Luis Polo ${ }^{1}$, Gina Polo L $^{2,3,4}$ \\ Facultad de Medicina Veterinaria y Zootecnia, Universidad Nacional de Colombia, Bogotá, D.C., Colombia \\ 2 Instituto Técnico de Educação e Controle Animal, Curitiba, Brasil \\ 3 Laboratorio de Epidemiologia e Bioestatística, Departamento de Medicina Veterinária y Preventiva e Saúde \\ Animal, Falculdade de Medicina Veterinária e Zootecnia, Universidade de São Paulo, São Paulo, Brasil \\ ${ }^{4}$ Grupo de Investigación en Epidemiología y Salud Pública, Universidad de La Salle, Bogotá, D.C., Colombia
}

Introducción. En Bogotá se desconoce el acceso que los habitantes tienen a los diferentes servicios de salud, lo que dificulta la planeación de las estrategias de prevención.

Objetivo. Estimar la accesibilidad espacial a los puestos de vacunación de la campaña antirrábica realizada en 2011 en Bogotá, y comparar la eficiencia de dos metodologías de estimación de la cobertura espacial.

Materiales y métodos. La accesibilidad espacial se determinó mediante el uso del modelo de área de influencia fluctuante de dos pasos (2SFCA). La cobertura espacial se calculó mediante la creación de zonas circulares de influencia utilizando distancias euclidianas y zonas irregulares aplicando el algoritmo de Dijkstra en las vías de la ciudad.

Resultados. La cobertura espacial del programa fue de $78,4 \%$ al usar las zonas circulares de influencia y de 60,2 \% con el algoritmo de Dijsktra. El análisis de accesibilidad espacial reveló que la zona norte de la ciudad y algunas zonas de la periferia presentaban menor accesibilidad al programa. Esta área periférica es una zona crítica por tratarse de una interfaz entre la zona rural y la urbana, lo cual representa un riesgo para la reaparición de la rabia en la ciudad.

Conclusiones. El modelo de accesibilidad espacial 2SFCA es una herramienta eficaz para identificar áreas aisladas, evaluar de forma más precisa el uso de los servicios de salud y proporcionar bases para su localización estratégica. Los resultados del análisis resaltan la necesidad de la vigilancia y la intervención en las áreas con bajo acceso a los servicios.

Palabras clave: rabia, vacunación, accesibilidad a los servicios de salud, perros, gatos.

doi: http://dx.doi.org/10.7705/biomedica.v36i3.3074

Assessment of the spatial accessibility to the rabies vaccination campaign in Bogotá, Colombia

Introduction: The access of Bogota's population to health services is unknown, and this hinders the planning of health prevention strategies.

Objective: To estimate the spatial accessibility to the vaccination sites of the 2011 campaign against rabies in Bogotá, Colombia, and to compare its efficiency with two other spatial coverage methodologies.

Materials and methods: Spatial accessibility was determined using the two-step floating catchment area model (2SFCA). We calculated spatial coverage by establishing circular buffer zones using Euclidean distances, and irregular zones around the vaccination sites using Dijkstra's algorithm on the city's street network.

Results: The spatial coverage of the program was $78.4 \%$ using the circular buffer zones, and $60.2 \%$ using Dijsktra's algorithm. The spatial accessibility analysis revealed that the periphery of the city had the lowest accessibility to the program. This peripheral area is a very critical zone because it is an urban-rural interface, which represents a risk for the re-introduction of rabies in the city.

Conclusions: The 2SFCA spatial accessibility model is an effective tool to identify isolated areas, evaluate health services use more precisely, and provide basis for their strategic location. We concluded that this approach had the potential to improve resource efficiency when planning rabies control programs in urban environments such as Bogotá. The findings emphasize the need for surveillance and intervention in isolated areas with low access to services.

Key words: rabies, vaccination, health services accessibility, dogs, cats. doi: http://dx.doi.org/10.7705/biomedica.v36i3.3074

\section{Contribución de los autores:}

Gina Polo: diseño del experimento

Stefany Monsalve, Daniel Santiago Rucinque, Gina Polo: realización del experimento y análisis de los datos

Todos los autores participaron en la escritura del manuscrito. 
Colombia cuenta con un "Plan estratégico para la eliminación de la rabia humana transmitida por el perro y el control de la rabia humana transmitida por murciélagos" desde el 2006 (1). Sin embargo, se continúan presentando casos de rabia en animales y humanos en todas las regiones del país. El último brote de rabia transmitida por perros fue ocasionado por la variante Ag1 del virus en Santa Marta entre el 2006 y el 2008, periodo en el que se presentaron cuatro casos en humanos y 28 casos en perros (2).

En Bogotá, la capital del país, las campañas de vacunación para perros y gatos han logrado controlar la incidencia de casos de rabia, a pesar de coberturas nacionales de vacunación antirrábica que oscilan entre 45 y $63 \%$ (3). En el 2007, el porcentaje de perros con evidencia serológica de vacunación contra el virus de la rabia en Bogotá fue de $24,5 \%$, por lo cual se concluyó que, en caso de un brote de la enfermedad causado por la inmigración de perros infectados en cualquier otra región del país, el virus podría transmitirse fácilmente entre la población canina y convertirse en un grave problema de salud pública (4).

A pesar de esto, desde 1982 no se han reportado casos de rabia humana en la capital y, desde el 2001, tampoco en animales (5). Debe anotarse, sin embargo, que en el 2012 se notificó un caso en una persona procedente de la región oeste del país en quien se confirmó la infección con la variante AgV4 del virus (6). En este contexto, el promover y garantizar el acceso a las campañas de vacunación en todos los grupos poblacionales debe ser una prioridad para los servicios de salud en todas las ciudades del país.

Para reubicar estratégicamente los puestos de vacunación contra la rabia ya existentes, o definir la localización de otros nuevos, es indispensable que previamente se determinen los grupos sociales y las áreas con poco acceso al servicio (7). La accesibilidad a los servicios se define como la facilidad relativa para llegar al lugar de prestación de un determinado servicio desde una determinada ubicación $(8,9)$, lo cual depende de factores como la disponibilidad de recursos, el tamaño de la

\footnotetext{
Correspondencia:

Gina Polo, Avenida Prof. Dr. Orlando Marques de Paiva, 87 CEP 05508 270, Cidade Universitária, São Paulo, Brasil Teléfono: (11) 30919937 y 30911393

gina@vps.fmvz.usp.br e imaginapolo@gmail.com

Recibido: 05/10/15; aceptado: 13/04/16
}

población, la distancia entre el lugar de prestación del servicio y la población, las barreras geográficas $\mathrm{y}$ el conocimiento que las personas tienen del servicio, entre otros (8-10).

Se han propuesto diversos métodos para estimar el acceso espacial a los servicios, entre los cuales pueden mencionarse el modelo de disponibilidad regional (11), el método de estimación de la densidad de Kernel (12), los modelos gravitacionales (13) y los modelos de área de influencia fluctuante (10). Estos últimos han sido los más empleados para evaluar el acceso a los servicios de salud humana $(8,14-17)$ y animal $(8,18)$.

En este estudio de evaluación de la accesibilidad espacial a los puestos de vacunación en la ciudad de Bogotá, se utilizó el método de área de influencia fluctuante de dos pasos (Two-Step Floating Catchment Area, 2SFCA). Las distancias se calcularon utilizando la red de transporte de la ciudad mediante el método del camino más corto y conforme al algoritmo de Dijkstra. Este método se comparó con el método clásico de análisis de cobertura espacial que se ha venido utilizando para evaluar el uso de diferentes servicios públicos.

Se espera que el estudio traiga beneficios directos a la comunidad al determinar áreas aisladas del programa de vacunación antirrábica para perros y gatos, y proporcionar bases para la reubicación estratégica de puestos de vacunación, lo que extrapolado podría contribuir, además, a la optimización de otros programas de salud en América Latina.

\section{Materiales y métodos \\ Área de estudio y bancos de datos}

El área de estudio fue la zona urbana de Bogotá que en el momento del estudio contaba con 7'347.795 habitantes (19), 1'725.792 domicilios (20), 684.858 perros y 144.525 gatos (21). Las direcciones de los 236 puestos de vacunación antirrábica en la campaña de octubre del 2011 se obtuvieron en el sitio en internet de la Secretaría Distrital de Salud (22). La georreferenciación de los puestos se hizo mediante el programa Batch Geocode ${ }^{\circledR}(23,24)$. Se georreferenciaron $218(90,8 \%)$ de los puestos de vacunación, ya que no se obtuvo la dirección de los 22 restantes (cuadro suplementario, disponible en: doi: http://dx.doi.org/10.7705/biomedica.v36i3.3074).

La distribución espacial de la población total de perros y gatos se estableció mediante la creación de puntos aleatorios seleccionados a partir del 
mapa de georreferenciación de los domicilios de la ciudad elaborado con el programa ArcGis $10.1^{\circledR}$. El número de perros y gatos se obtuvo a partir de estimaciones previas de su población por localidades (21); la aleatoriedad fue una consecuencia de la falta de conocimiento de la ubicación real de la población animal.

\section{Cálculo de la cobertura espacial del programa} de vacunación antirrábica

Para calcular la cobertura espacial de los puestos de vacunación antirrábica en la ciudad, se usaron dos metodologías. La primera consistió en la creación de zonas circulares de influencia alrededor de los puestos de vacunación utilizando distancias euclidianas, y la segunda, en el establecimiento de zonas irregulares alrededor de los puestos de vacunación mediante el uso de la red de transporte de la ciudad utilizando el algoritmo de Dijkstra, el cual se basa en el método del camino más corto. En ambos casos se determinó un radio de $800 \mathrm{~m}$, distancia de desplazamiento considerada razonable para el traslado a pie de una persona en busca de un servicio de salud $(8,23)$.

La proporción de la población cubierta por cada una de estas dos áreas de servicio se comparó mediante la prueba de ji al cuadrado. Los valores de las distancias de desplazamiento calculadas con el modelo de zonas de influencia circulares y con zonas irregulares hasta el puesto de vacunación más cercano se determinaron a lo largo de la red de transporte y se compararon usando el test de Wilcoxon. Los análisis se hicieron con el programa R, versión 2.14.0, y un nivel de significación de $5 \%$.

\section{Cálculo de la accesibilidad espacial al programa de vacunación antirrábica}

Para determinar la accesibilidad espacial, se utilizó el modelo de área de influencia fluctuante de dos pasos (2SFCA) basado en una función gaussiana con coeficiente de fricción $(8,9,18,25)$. El primer paso del modelo 2SFCA consistió en generar para cada puesto de vacunación, $j$, un área de servicio delimitada por una distancia límite, $d_{0}$. Posteriormente, se ubicó toda la población $k$ dentro de la distancia $d_{0}$ y se asignó a cada $k$ un peso de Gauss, $G$, para computar, así, la razón entre servicio y población, $R_{j}$, de cada puesto de vacunación:

$$
R_{j}=\frac{S_{j}}{\sum_{k \in\left(d_{k j} \leq d_{0}\right)} P_{k} G(d k j, d 0)},
$$

donde $S_{j}$ es la capacidad, representada por las dosis de vacunación de cada puesto de vacunación $j, P_{k}$ es la población $k$ dentro del área de servicio $d_{0}, d_{k j}$ es la distancia entre la población $k$ y los puestos de vacunación $j$, y $G$ corresponde al peso de Gauss determinado por:

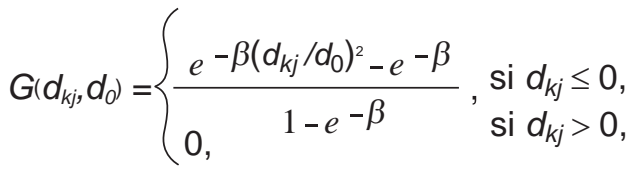

donde $\beta$ es el coeficiente de fricción que representa la facilidad de desplazamiento a pie en condiciones de carretera $(8,18)$. En este estudio se usó un valor de 0,5 por haberse determinado en estudios dinámicos previos como el valor adecuado para representar la facilidad de desplazamiento a pie en áreas urbanas (26).

El segundo paso consistió en generar un área de servicio para cada domicilio, $i$, y buscar todos los puestos de vacunación, I, dentro del área determinada por $d_{0}$, asignando un peso de Gauss diferente a cada puesto. Después se computaron estos pesos con las razones entre servicio y población para obtener la accesibilidad espacial, $A_{i}$, de cada domicilio de la población, $i$ :

$$
A_{i}=\Sigma_{k \in\left(d_{k j} \leq d_{0}\right)} R_{j} G\left(d_{i l}, d_{0}\right),
$$

donde / se refiere a todos los puestos de vacunación dentro del área de servicio de la población $i$; todas las demás anotaciones son las mismas que en la ecuación 1. La figura 1 muestra un diagrama de flujo de la metodología descrita.

\section{Resultados}

En la campaña de vacunación antirrábica de octubre de 2011 en Bogotá, se distribuyeron 240 puestos en los que se aplicaron 388.157 vacunas, con 1.617 dosis aplicadas por puesto (27). La distribución espacial de los puestos de vacunación antirrábica con respecto a la densidad de la población de perros y gatos se muestra en la figura 2, en la cual puede observarse que la mayor parte de los puestos de vacunación se encontraba en la zona suroeste de la ciudad, donde había una mayor densidad de gatos y perros. Asimismo, se observó una menor cantidad de puestos de vacunación en la zona norte de la ciudad, que, aunque no tenía la mayor cantidad de gatos y perros, sí contaba con algunas zonas en las que la densidad de la población de estos animales era alta o media.

Se calculó que la cobertura espacial del programa fue de $78,4 \%$ al usar las zonas circulares de influencia y de $60,2 \%$ con las distancias a lo largo 


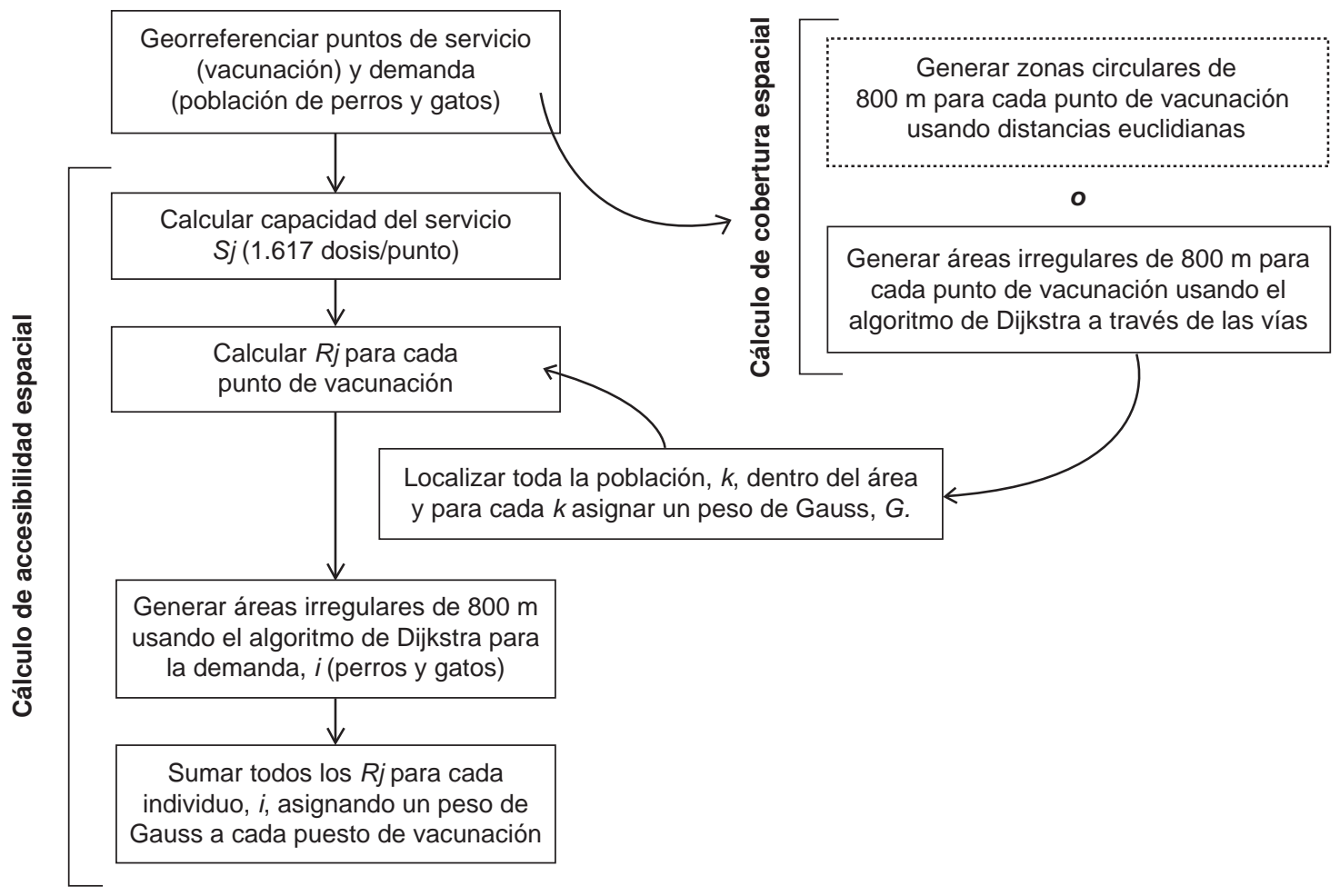

Figura 1. Diagrama de flujo de la metodología descrita

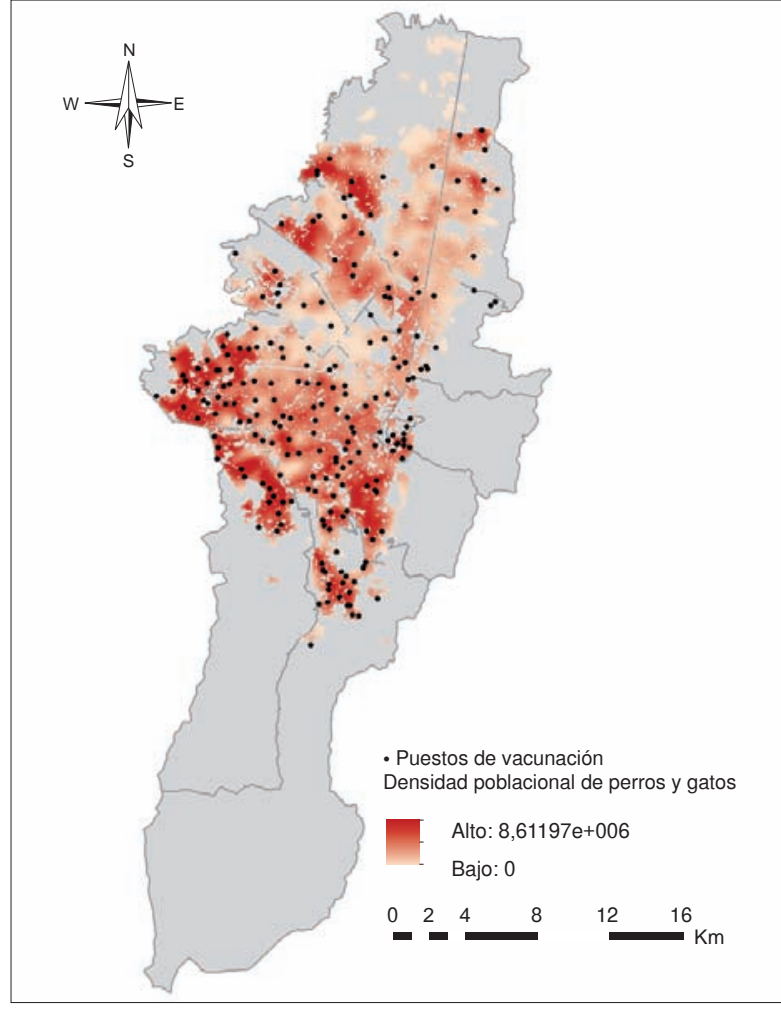

Figura 2. Distribución espacial de los puestos de vacunación antirrábica en relación con la densidad poblacional de perros y gatos en Bogotá de la red de transporte de la ciudad (figura 3); en ambas situaciones hubo una mayor cobertura en la zona sur de la ciudad.

Así, el número de animales bajo cobertura del programa de vacunación fue significativamente mayor $(p<0,05)$ en las áreas de servicio calculadas mediante las distancias euclidianas (lineales) que en aquellas creadas mediante el algoritmo de Dijkstra a lo largo de la red de transporte. La distancia media derivada del camino más corto a través de la red de transporte para propietarios de perros fue de 562,53 m en las áreas circulares y de 469,83 m en las áreas irregulares derivadas del algoritmo de Dijkstra. Para los propietarios de gatos la distancia media fue de 560,71 m en las áreas circulares y de $468,83 \mathrm{~m}$ en las áreas derivadas del algoritmo de Dijkstra.

Se encontró que el desplazamiento de las personas con perros y gatos fue mayor en las áreas circulares $(p<0,05)$. En la figura 4 se muestra la accesibilidad espacial para los propietarios de perros y gatos de los puestos de vacunación establecidos durante la campaña de vacunación antirrábica en Bogotá durante el 2011, determinada mediante el método 2SFCA con un coeficiente de fricción de transporte basado en una función gaussiana. 

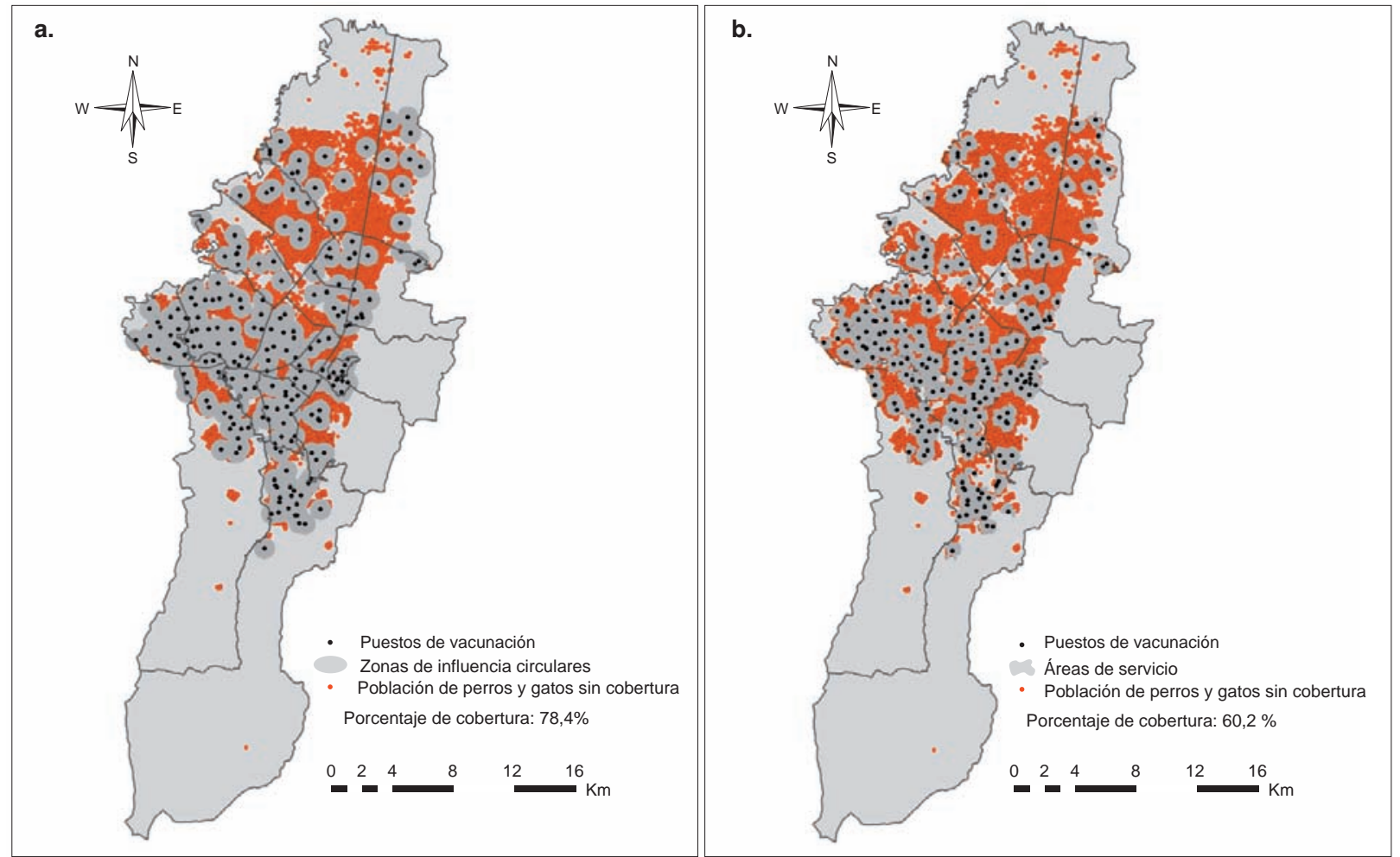

Figura 3. Cobertura espacial del programa de vacunación antirrábica representada mediante a) zonas de influencia circulares calculadas con distancias euclideanas, y b) zonas irregulares calculadas con el algoritmo de Dijkstra

Puede observarse una gran área de poca accesibilidad $(0,0001>0,1)$ y de accesibilidad espacial nula $(0,0)$ en la zona norte de la ciudad, en donde se observan focos de gran accesibilidad $(>0,2)$ coincidentes con áreas de baja densidad poblacional. La parte central de la ciudad, de baja densidad poblacional, presentó zonas de gran accesibilidad espacial $(>0,2)$. En la zona suroeste de la ciudad, que correspondía a la más densamente poblada, se encontró la mayor cantidad de puestos, por lo que allí se presentó una accesibilidad espacial media $(0,1>0,2)$. La periferia de la ciudad presentó poca $(0,0001>0,1)$ y nula $(0,0)$ accesibilidad en algunas áreas; esta podría considerarse como una zona de riesgo de rabia, pues se trata de un área en que limitan la zona rural y la urbana, y a la cual ingresan en mayor medida personas y animales desde zonas rurales.

Igualmente, se evidenció que la metodología clásica de análisis de cobertura se ha sobrestimado frente a la metodología de accesibilidad espacial, pues la primera no revela plenamente la situación del programa al omitir variables como el tamaño poblacional, la distancia, la disponibilidad de varios puntos o el diseño de la red de transporte.

\section{Discusión}

Dado el riesgo de presentación de casos de rabia en Bogotá, el objeto del presente estudio fue contribuir a la planeación espacial del programa de vacunación antirrábica para garantizar un mejor acceso de la población a sus servicios.

Para ello, se evaluó la accesibilidad de los propietarios de perros y gatos a la campaña de vacunación antirrábica de octubre de 2011 en la ciudad, utilizando el método de área fluctuante de dos pasos (2SFCA) basado en una función gaussiana (9) y con un coeficiente de fricción de transporte que representó la facilidad de traslado a pie de un usuario promedio en condiciones de carretera (8). Se utilizó una distancia de $800 \mathrm{~m}$, considerada como razonable para recorrerla a pie en busca de un servicio de salud (23), a la cual se recurrió debido a la falta de información sobre la distancia promedio que una persona estaría dispuesta a caminar para llevar su animal de compañía hasta un puesto de vacunación.

El método consistente en calcular zonas de influencia circulares alrededor de los lugares de prestación de servicios se ha utilizado ampliamente 


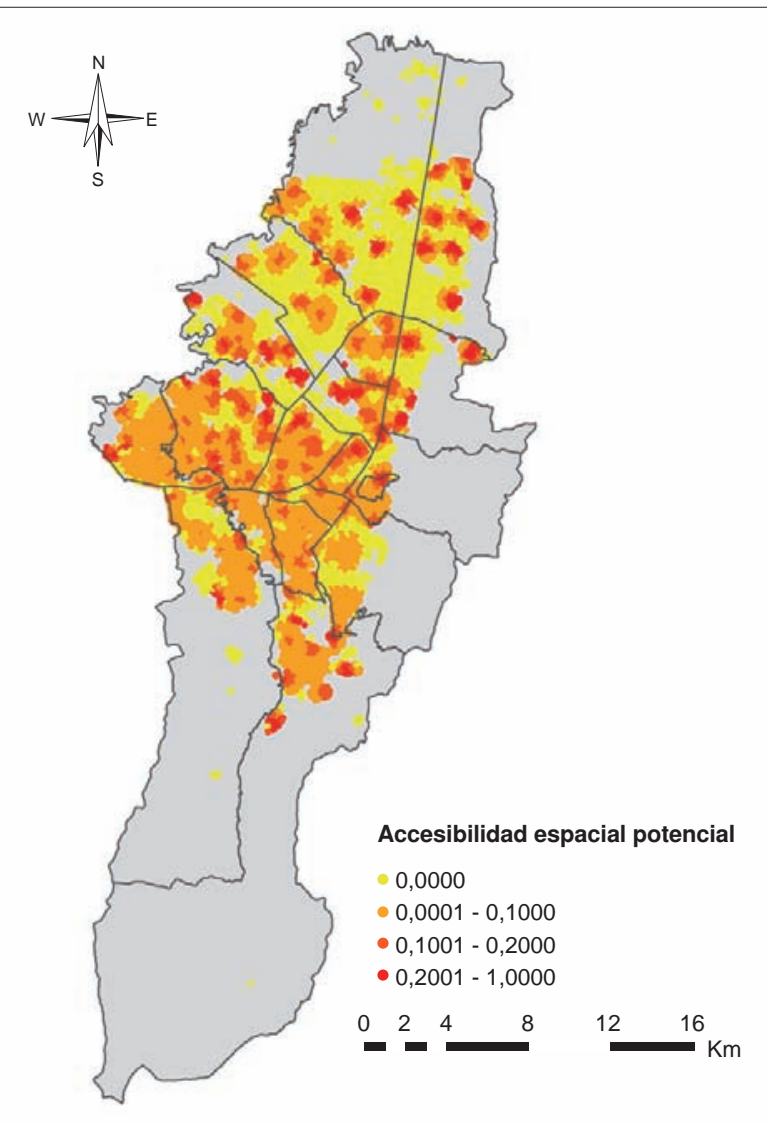

Figura 4. Accesibilidad espacial potencial de los propietarios de perros y gatos a los puestos de vacunación establecidos durante la campaña de vacunación antirrábica realizada en Bogotá durante el 2011 y calculada con el método 2SFCA

en estudios de accesibilidad debido a la facilidad de su cálculo (28), asumiendo que la distancia recorrida por una persona que se transporta hasta un determinado servicio es la misma obtenida en el cálculo de la distancia euclidiana. No obstante, la distancia de desplazamiento a través de la red de transporte es mayor, como se evidenció en este trabajo, debido a que el desplazamiento por las calles sigue su diseño en vez del avance en línea recta.

De esta forma, el análisis de cobertura que contempla zonas circulares (distancia en línea recta) tiende a sobreestimar el área de cobertura comparado con aquel que contempla zonas irregulares basadas en el método del camino más corto a lo largo de la red de transporte. Sin embargo, ninguno de estos análisis de cobertura revela el uso de un determinado servicio, ya que omite variables como el tamaño poblacional, la capacidad del servicio o las barreras geográficas.
En consecuencia, a diferencia de los análisis de cobertura, los análisis de accesibilidad revelan el uso de un determinado servicio con mayor precisión. Mediante el análisis de accesibilidad espacial, en este estudio se determinó que había poca accesibilidad al servicio de vacunación antirrábica en Bogotá. Las zonas de mayor accesibilidad correspondieron a aquellas con baja densidad poblacional y alto número de puestos de vacunación.

Por ser una ciudad con un elevado flujo de personas y animales, Bogotá está en riesgo constante de presentar casos de rabia urbana transmitida por perros o gatos infectados provenientes de distintas partes del país. Este trabajo aspira a contribuir a orientar las estrategias de prevención en las zonas aisladas del servicio con alta densidad poblacional.

En este estudio se utilizó un modelo para peatones, pero otros modelos de transporte por medio de automóviles particulares y transporte público pueden dar una visión más completa del acceso a los diferentes servicios. El diseño de un modelo para peatones permite un análisis más preciso, ya que es independiente del tráfico y del sentido de las vías. Aunque los aspectos espaciales sean fundamentales para una adecuada planeación de los servicios, se requieren estudios que evalúen otros factores no espaciales, como el nivel socioeconómico o el conocimiento de las personas del servicio, para complementar los análisis y mejorar la planeación del programa de vacunación.

Es importante destacar que en el presente estudio no se contempló el acceso a los servicios privados, aspecto que podría complementar otros estudios orientados a determinar áreas de riesgo para la presentación de casos de rabia. Además, tampoco se consideró el peso que puede tener la proximidad de una persona a más de un punto de vacunación (18).

Se demostró que la estimación de la accesibilidad espacial es una herramienta eficaz que debería utilizarse en la planeación y evaluación de diferentes programas de salud. Los resultados del análisis sustentan la necesidad de vigilancia e intervención en las áreas aisladas del servicio.

\section{Conflicto de intereses}

Los autores manifiestan que no tienen conflicto de intereses con respecto al manuscrito.

\section{Financiación}

Los autores no recibieron financiación para el desarrollo de este trabajo. 


\section{Referencias}

1. Cediel-Becerra NM. Asociación entre la epidemiología de la rabia canina, la organización del programa de rabia y las condiciones socio-económicas en Colombia. Estudio ecológico (tesis). Bogotá: Universidad Nacional de Colombia; 2009.

2. Páez A, Rey G, Agudelo C, Dulce A, Parra E, DíazGranados $\mathbf{H}$, et al. Brote de rabia urbana transmitida por perros en el distrito de Santa Marta, Colombia, 20062008. Biomédica. 2009;29:424-36. http://dx.doi.org/10.7705/ biomedica.v29i3.14

3. Cediel N, de la Hoz F, Villamil LC, Romero J, Díaz A. Epidemiología de la rabia canina en Colombia. Rev Salud Pública. 2010;12:368-79. http://dx.doi.org/10.1590/S012400642010000300003

4. Páez A, Meza J, Calvo P, Garzón P. Niveles de inmunidad humoral conferidos con la primera dosis de la vacuna antirrábica en caninos con dueño de la ciudad de Bogotá, Colombia. Revista de Investigación. 2007;7:191-7.

5. Secretaría Distrital de Salud. Jornada de vacunación canina en Bogotá. 2008. Fecha de consulta: 8 de enero de 2013. Disponible en: http://www.saludcapital.gov.co/Lists/ Anuncionprincipales/DispForm.aspx?ID=65

6. Walteros DM, Castro Á. Rabia humana y rabia animal, situación actual en Colombia hasta el periodo epidemiológico décimo de 2012. Informe de evento. Bogotá: Instituto Nacional de Salud; 2012. p. 1-29. Fecha de consulta: 8 de enero de 2013. Disponible en: http://www.ins.gov.co/ lineas-de-accion/Subdireccion-Vigilancia/Informe de Evento Epidemiolgico/RABIA 2012.pdf

7. Wan N, Zou B, Sternberg T. A three-step floating catchment area method for analyzing spatial access to health services. Int J Geogr Inf Sci. 2012;26:1073-89. http://dx.doi.org/10.10 80/13658816.2011.624987

8. Polo G, Acosta CM, Dias RA. Spatial accessibility to vaccination sites in a campaign against rabies in São Paulo city, Brazil. Prev Vet Med. 2013;111:10-6. http://dx.doi. org/10.1016/j.prevetmed.2013.03.010

9. Dai D. Racial/ethnic and socioeconomic disparities in urban green space accessibility: Where to intervene? Landsc Urban Plan. 2011;102:234-44. http://dx.doi.org/10.1016/j. landurbplan.2011.05.002

10. Luo W, Wang F. Measures of spatial accessibility to health care in a GIS environment: Synthesis and a case study in the Chicago region. Environ Plann B Plann Des. 2003;30:86584. http://dx.doi.org/10.1068/b29120

11. Khan A. An integrated approach to measuring potential spatial access to health care services. Socioecon Plann Sci. 1992;26:275-87. http://dx.doi.org/10.1016/0038-0121 (92) $90004-\mathrm{O}$

12. Guagliardo MF. Spatial accessibility of primary care: Concepts, methods and challenges. Int J Health Geogr. 2004;3:1-13. http://dx.doi.org/10.1186/1476-072X-3-3

13. Joseph A, Bantock P. Measuring potential physical accessibility to general practitioners in rural areas: A method and case study. Soc Sci Med. 1982;16:85-90. http://dx.doi. org/10.1016/0277-9536(82)90428-2
14. Langford M, Higgs G. Measuring potential access to primary healthcare services: The influence of alternative spatial representations of population. Prof Geogr. 2006;58:294-306. http://dx.doi.org/10.1111/j.1467-9272.2006.00569.x

15. Wang L. Immigration, ethnicity, and accessibility to culturally diverse family physicians. Health Place. 2007;13:656-71. http://dx.doi.org/10.1016/j.healthplace.2006.10.001

16. Wang F, McLafferty S, Escamilla V, Luo L. Latestage breast cancer diagnosis and health care access in Illinois. Prof Geogr. 2008;60:54-69. http://dx.doi.org/10. $1080 / 00330120701724087$

17. Yang D-H, Goerge R, Mullner R. Comparing GIS-based methods of measuring spatial accessibility to health services. J Med Sys. 2006;30:23-32. http://dx.doi.org/10. 1007/s10916-006-7400-5

18. Polo G, Mera-Acosta C, Ferreira F, Dias RA. Locationallocation and accessibility models for improving the spatial planning of public health services. PLoS One. 2015;16:114. http://dx.doi.org/10.1371/journal.pone.0119190

19. Departamento Administrativo Nacional de Estadística. Boletín Censo General 2005. Perfil Bogotá. Bogotá: DANE; 2010. Fecha de consulta: 19 de enero de 2013. Disponible en: http://www.dane.gov.co/files/censo2005/PERFIL_PDF_ CG2005/11001T7T000.PDF

20. Infraestructura de Datos Espaciales para el Distrito Capital (IDECA). Catálogo de Datos Geográficos, Nomenclatura Domiciliaria. Bogotá: IDECA; 2012.

21. Vega-Aragón RL, Espinosa-Garzón GH, Castillo-Bejarano L. Análisis de la población canina en el distrito capital, 2005. Investig Segur Soc Salud. 2007;9:205-27.

22. Secretaría Distrital de Salud. Gran jornada de vacunación y adopción de perros y gatos. 2011. Fecha de consulta: 10 de enero de 2013. Disponible en: http://www.saludcapital. gov.co/Lists/Anuncionprincipales/DispForm.aspx?ID=533

23. Bell S, Wilson K, Shah TI, Gersher S, Elliott T. Investigating impacts of positional error on potential health care accessibility. Spat Spatiotemporal Epidemiol. 2012;3:17-29. http:// dx.doi.org/ 10.1016/j.sste.2012.02.003

24. Zwiefelhofer D. Batch Geocoding. 2012. Fecha de consulta: 2 de diciembre de 2012. Disponible en: http:// www.findlatitudeandlongitude.com/batch-geocode/\#.Vw6k PnhDIU.

25. Dai D. Black residential segregation, disparities in spatial access to health care facilities, and late-stage breast cancer diagnosis in metropolitan Detroit. Health Place. 2010;16:1038-52. http://dx.doi.org/10.1016/j.healthplace. 2010.06.012

26. Marpet ML. On threshold values that separate pedestrian walkways that are slip resistant from those that are not. J Forensic Sci. 1996;41:747-55. http://dx.doi.org/10.1520/ JFS13992J

27. Secretaría Distrital de Ambiente. Plan Institucional de Gestión Ambiental - PIGA. Consulta de indicadores ambientales - 2012. Fecha de consulta: 10 de enero de 2013. Disponible en: http://ambientebogota.gov.co/ko/planinstitucional-de-gestion-ambiental-piga1

28. Gutiérrez J, García-Palomares JC. Distance-measure impacts on the calculation of transport service areas using GIS. Environ Plann B. 2008;35:480-503. http://dx.doi.org/10. 1068/b33043 
Cuadro suplementario. Latitud y longitud de los puntos de vacunación de la campaña de vacunación antirrábica para perros y gatos en Bogotá, 2012

\begin{tabular}{|c|c|c|c|c|c|}
\hline ID & Latitud & Longitud & ID & Latitud & Longitud \\
\hline 1 & 4,6724668000 & $-74,1525397000$ & 75 & 4,5189016000 & $-74,0931380000$ \\
\hline 2 & 4,6871367000 & $-74,1456129000$ & 76 & 4,6025406000 & $-74,1036871000$ \\
\hline 3 & 4,6672416000 & $-74,1433528000$ & 77 & 4,5662231000 & $-74,0869186000$ \\
\hline 4 & 4,6791217000 & $-74,1427383000$ & 78 & 4,6245236000 & $-74,0687646000$ \\
\hline 5 & 4,6747362000 & $-74,1437547000$ & 79 & 4,5484670000 & $-74,1041149000$ \\
\hline 6 & 4,6675031000 & $-74,1290404000$ & 80 & 4,6368508000 & $-74,0770542000$ \\
\hline 7 & 4,6742613000 & $-74,1443323000$ & 81 & 4,5819214000 & $-74,1317919000$ \\
\hline 8 & 4,6691662000 & $-74,1187784000$ & 82 & 4,5791449000 & $-74,1103301000$ \\
\hline 9 & 4,6555855000 & $\begin{array}{l}-74,1132725000 \\
-741683861000\end{array}$ & 83 & 4,5613362000 & $-74,1262421000$ \\
\hline 10 & & $\begin{array}{l}-74,1683861000 \\
-74,1478379000\end{array}$ & 84 & 4,5809588000 & $-74,1384215000$ \\
\hline $\begin{array}{l}11 \\
12\end{array}$ & $\begin{array}{l}4,6222634000 \\
4,6223392000\end{array}$ & $-74,1636567000$ & 85 & 4,6140350000 & $-74,0876016000$ \\
\hline 13 & 4,6126492000 & $-74,1461219000$ & 86 & 4,5901153000 & $-74,1375668000$ \\
\hline 14 & 4,6234627000 & $-74,1575012000$ & 87 & 4,5879377000 & $-74,1473021000$ \\
\hline 15 & 4,6122354000 & $-74,1570401000$ & 88 & 4,5892694000 & $-74,1527761000$ \\
\hline 16 & 4,6019577000 & $-74,1365768000$ & 89 & 4,5165202000 & $-74,0945161000$ \\
\hline 17 & 4,6041217000 & $-74,1561227000$ & 90 & 4,4979549000 & $-74,0863657000$ \\
\hline 18 & 4,5974351000 & $-74,1427813000$ & 91 & 4,5246465000 & $-74,1097719000$ \\
\hline 19 & 4,6036331000 & $-74,1410330000$ & 92 & 4,5247570000 & $-74,1099986000$ \\
\hline 20 & 4,5997123000 & $-74,1458254000$ & 93 & 4,5131729000 & $-74,1165583000$ \\
\hline 21 & 4,6372363000 & $-74,1410270000$ & 94 & 4,5402450000 & $-74,1173103000$ \\
\hline 22 & 4,6427154000 & $-74,1418906000$ & 95 & 4,5377614000 & $-74,1140342000$ \\
\hline 23 & 4,6458096000 & $-74,1479657000$ & 96 & 4,5182748000 & $-74,1182502000$ \\
\hline 24 & $\begin{array}{l}4,6232877000 \\
46325090000\end{array}$ & $\begin{array}{l}-74,1321934000 \\
-74,1272738000\end{array}$ & 97 & 4,4947861000 & $-74,1200755000$ \\
\hline $\begin{array}{l}25 \\
26\end{array}$ & $\begin{array}{l}4,6325090000 \\
4,6222799000\end{array}$ & $\begin{array}{l}72738000 \\
69530000\end{array}$ & 98 & 4,4957344000 & $-74,1152509000$ \\
\hline $\begin{array}{l}26 \\
27\end{array}$ & $\begin{array}{l}4,6222799000 \\
4,6425906000\end{array}$ & $-74,1261563000$ & 99 & 4,4986968000 & $-74,1084919000$ \\
\hline 28 & 4,6540528000 & $-74,1570430000$ & 100 & 4,5060594000 & $-74,1143784000$ \\
\hline 29 & 4,6433329000 & $-74,1560916000$ & 101 & 4,5030201000 & $-74,1146095000$ \\
\hline 30 & 4,6424875000 & $-74,1607093000$ & 102 & 4,4710460000 & $-74,1250799000$ \\
\hline 31 & 4,6505940000 & $-74,1732623000$ & 103 & 4,5071398000 & $-74,1063510000$ \\
\hline 32 & 4,6425765000 & $-74,1658072000$ & 104 & 4,5142288000 & $-74,1176762000$ \\
\hline 33 & 4,6304452000 & $-74,1709549000$ & 105 & 4,5132616000 & $-74,1072679000$ \\
\hline 34 & 4,6298037000 & $-74,1777181000$ & 106 & 4,5107820000 & $-74,1047947000$ \\
\hline 35 & 4,6390501000 & $-74,1728207000$ & 107 & 4,5075717000 & $-74,0998009000$ \\
\hline 36 & 4,6438477000 & $-74,1755776000$ & 108 & 4,4883575000 & $-74,1010438000$ \\
\hline 37 & 4,6143066000 & -74,1783773000 & 109 & 4,4878664000 & $-74,0971491000$ \\
\hline 38 & 4,6218964000 & $-74,1717304000$ & 110 & 4,4937947000 & $-74,1038981000$ \\
\hline 39 & 4,6207725000 & $-74,1751516000$ & 111 & 4,5015110000 & $-74,1026858000$ \\
\hline 40 & 4,6105232000 & $-74,1692944000$ & 112 & 4,4938967000 & $-74,1024592000$ \\
\hline 41 & 4,6103778000 & $-74,1739946000$ & 113 & 4,7575069000 & $-74,0243189000$ \\
\hline 42 & 4,6161922000 & $-74,1048878000$ & 114 & 4,7688981000 & $-74,0260920000$ \\
\hline 43 & 4,6221431000 & $-74,1204798000$ & 115 & 4,7344613000 & $-74,0172735000$ \\
\hline 44 & 4,6199071000 & $-74,1120089000$ & 116 & 4,7659939000 & $-74,0391184000$ \\
\hline 45 & 4,6103336000 & $-74,1186693000$ & 117 & 4,7394911000 & $-74,0400904000$ \\
\hline 46 & 4,6076493000 & $-74,1098598000$ & 118 & 4,7215575000 & $-74,0302332000$ \\
\hline 47 & 4,6025205000 & 74,1079128000 & 119 & 4,7215575000 & $-74,0302332000$ \\
\hline 48 & 4,6208136000 & $-74,1051798000$ & 120 & 4,7397642000 & $-74,0249220000$ \\
\hline 49 & 4,6300125000 & $-74,1142116000$ & 121 & 4,6956637000 & $-74,0310243000$ \\
\hline 50 & 4,6084299000 & $-74,1232579000$ & 122 & 4,6184112000 & $-74,1899698000$ \\
\hline 51 & 4,6009442000 & $-74,1224670000$ & 123 & 4,7145832000 & $-74,1419160000$ \\
\hline 52 & 4,6324492000 & $-74,1109822000$ & 124 & 4,7188319000 & $-74,1054839000$ \\
\hline 53 & 4,7417665000 & $-74,0833268000$ & 125 & 4,6937781000 & $-74,1109452000$ \\
\hline 54 & 4,7479078000 & $-74,0548182000$ & 126 & 4,6725604000 & $-74,0821990000$ \\
\hline 55 & 4,7248253000 & $-74,0703953000$ & 127 & 4,6845935000 & $-74,1006076000$ \\
\hline 56 & 4,6970468000 & $-74,0758580000$ & 128 & 4,7191320000 & $-74,1200017000$ \\
\hline 57 & 4,7307754000 & $-74,1020913000$ & 129 & 4,7164463000 & $-74,1235720000$ \\
\hline 58 & 4,7198789000 & $-74,0902684000$ & 130 & 4,6778714000 & $-74,0801846000$ \\
\hline 59 & 4,7298367000 & $-74,1057656000$ & 131 & 4,6909575000 & $-74,0999589000$ \\
\hline 60 & 4,7092893000 & $-74,0954168000$ & 132 & 4,5883975000 & $-74,1034968000$ \\
\hline 61 & 4,7389183000 & $-74,1011635000$ & 133 & 4,5938098000 & $-74,1002008000$ \\
\hline 62 & 4,7455031000 & $-74,1213415000$ & 134 & 4,5823620000 & $-74,0973640000$ \\
\hline 63 & 4,7522339000 & $-74,1139947000$ & 135 & 4,5863442000 & $-74,0877868000$ \\
\hline 64 & 4,7431653000 & $-74,1212037000$ & 136 & 4,5934914000 & $-74,1232844000$ \\
\hline 65 & 4,5931847000 & $-74,0713958000$ & 137 & 4,5766085000 & $-74,1021530000$ \\
\hline 66 & 4,5368014000 & $-74,0839008000$ & 138 & 4,5765701000 & $-74,1103712000$ \\
\hline 67 & 4,6674006000 & $-74,0209975000$ & 139 & 4,5840666000 & $-74,1050795000$ \\
\hline 68 & 4,5604392000 & $-74,0878396000$ & 140 & 4,5832037000 & $-74,1197183000$ \\
\hline 69 & 4,5630412000 & $-74,0923870000$ & 141 & 4,5734183000 & $-74,1062738000$ \\
\hline 70 & 4,5591883000 & $-74,0870368000$ & 142 & 4,5636559000 & $-74,1065742000$ \\
\hline 71 & 4,5156971000 & $-74,0947588000$ & 143 & 4,5690049000 & $-74,1096619000$ \\
\hline 72 & 4,5319548000 & $-74,0893391000$ & 144 & 4,5573481000 & $-74,1118956000$ \\
\hline 73 & 4,5683943000 & $-74,1235372000$ & 145 & 4,5479788000 & $-74,1134667000$ \\
\hline 74 & 4,5369691000 & $-74,0925713000$ & 146 & 4,5432096000 & $-74,1179779000$ \\
\hline
\end{tabular}

\begin{tabular}{|c|c|c|}
\hline ID & Latitud & Longitud \\
\hline 147 & 4,5599265000 & $-74,1224301000$ \\
\hline 148 & 4,5452884000 & $-74,1063889000$ \\
\hline 149 & 4,5672916000 & $-74,1160743000$ \\
\hline 150 & 4,6530582000 & $-74,0736973000$ \\
\hline 151 & 4,6238311000 & $-74,1970709000$ \\
\hline 152 & 4,6000987000 & $-74,1656889000$ \\
\hline 153 & 4,6083171000 & $-74,1984086000$ \\
\hline 154 & 4,6270703000 & $-74,1987243000$ \\
\hline 155 & 4,6177857000 & $-74,2042117000$ \\
\hline 156 & 4,6363837000 & $-74,2042976000$ \\
\hline 157 & 4,6308107000 & $-74,1899259000$ \\
\hline 158 & 4,6148009000 & $-74,2139138000$ \\
\hline 159 & 4,6020937000 & $-74,1903048000$ \\
\hline 160 & 4,6123394000 & $-74,1868242000$ \\
\hline 161 & 4,6298860000 & $-74,1792314000$ \\
\hline 162 & 4,6006754000 & $-74,1603283000$ \\
\hline 163 & 4,6222936000 & $-74,1844927000$ \\
\hline 164 & 4,6107679000 & $-74,1842926000$ \\
\hline 165 & 4,6358763000 & $-74,1846072000$ \\
\hline 166 & 4,6046484000 & $-74,1800361000$ \\
\hline 167 & 4,5973197000 & $-74,0695034000$ \\
\hline 168 & 4,5924254000 & $-74,0774659000$ \\
\hline 169 & 4,6018465000 & $-74,0675973000$ \\
\hline 170 & 4,7233993000 & $-74,0463274000$ \\
\hline 171 & 4,5970644000 & $-74,1011821000$ \\
\hline 172 & 4,6090966000 & $-74,0932165000$ \\
\hline 173 & 4,6161253000 & $-74,0807954000$ \\
\hline 174 & 4,6256284000 & $-74,0659470000$ \\
\hline 175 & 4,5898344000 & $-74,0713869000$ \\
\hline 176 & 4,5853557000 & $-74,0677659000$ \\
\hline 177 & 4,5789023000 & $-74,0720307000$ \\
\hline 178 & 4,5866916000 & $-74,0713960000$ \\
\hline 179 & 4,5879438000 & $-74,0745537000$ \\
\hline 180 & 4,5833486000 & $-74,0775195000$ \\
\hline 181 & 4,5870884000 & $-74,0753925000$ \\
\hline 182 & 4,5891983000 & $-74,0803630000$ \\
\hline 183 & 4,5942050000 & $-74,0849794000$ \\
\hline 184 & 4,6317994000 & $-74,0741782000$ \\
\hline 185 & 4,6461045000 & $-74,0906715000$ \\
\hline 186 & 4,6480861000 & $-74,0820051000$ \\
\hline 187 & 4,6481132000 & $-74,0823195000$ \\
\hline 188 & 4,6618904000 & $-74,0904448000$ \\
\hline 189 & 4,6718812000 & $-74,0792756000$ \\
\hline 190 & 4,6826965000 & $-74,0643857000$ \\
\hline 191 & 4,6748249000 & $-74,0628155000$ \\
\hline 192 & 4,6711106000 & $-74,0666098000$ \\
\hline 193 & 4,6307023000 & $-74,0576048000$ \\
\hline 194 & 4,6428937000 & $-74,0526230000$ \\
\hline 195 & 4,6304030000 & $-74,0612637000$ \\
\hline 196 & 4,6324726000 & $-74,0583265000$ \\
\hline 197 & 4,6760226000 & $-74,0307297000$ \\
\hline 198 & 4,6693475000 & $-74,0184664000$ \\
\hline 199 & 4,6730409000 & $-74,0537864000$ \\
\hline 200 & 4,6496618000 & $-74,0634654000$ \\
\hline 201 & 4,6342967000 & $-74,0705339000$ \\
\hline 202 & 4,5370380000 & $-74,1444008000$ \\
\hline 203 & 4,5535677000 & $-74,1410323000$ \\
\hline 204 & 4,5569036000 & $-74,1464697000$ \\
\hline 205 & 4,5390569000 & $-74,1548784000$ \\
\hline 206 & 4,5615755000 & $-74,1485479000$ \\
\hline 207 & 4,5645257000 & $-74,1527550000$ \\
\hline 208 & 4,5470054000 & $-74,1434217000$ \\
\hline 209 & 4,5534087000 & $-74,1487930000$ \\
\hline 210 & 4,5691966000 & $-74,1426984000$ \\
\hline 211 & 4,5925742000 & $-74,1568232000$ \\
\hline 212 & 4,5853456000 & $-74,1784329000$ \\
\hline 213 & 4,5913812000 & $-74,1805955000$ \\
\hline 214 & 4,5542447000 & $-74,1362247000$ \\
\hline 215 & 4,5684260000 & $-74,1632924000$ \\
\hline 216 & 4,5786531000 & $-74,1787945000$ \\
\hline 217 & 4,5404264000 & $-74,1420664000$ \\
\hline 218 & 4,5727678000 & $-74,1649050000$ \\
\hline
\end{tabular}

